\title{
Towards a Framework for Validating the Matching Between Notifications and Scents in Olfactory In-Car Interaction
}

\author{
Dmitrijs Dmitrenko, Emanuela Maggioni, Marianna Obrist \\ \{d.dmitrenko,e.maggioni,m.obrist\}@sussex.ac.uk \\ SCHI Lab, Creative Technology Research Group, \\ School of Engineering and Informatics, University of Sussex \\ Brighton, United Kingdom
}

\begin{abstract}
Olfactory notifications have been proven to have a positive impact on drivers. This has motivated the use of scents to convey driving-relevant information. Research has proposed the use of such scents as lemon, peppermint, lavender and rose for in-car notifications. However, there is no framework to identify which scent is the most suitable for every application scenario. In this paper, we propose an approach for validating a matching between scents and driving-relevant notifications. We suggest a study in which the olfactory modality is compared with a puff of clean air, visual, auditory, and tactile stimuli while performing the same driving task. For the data analysis, we suggest recording the lane deviation, speed, time required to recover from the error, as well as the perceived liking and comfort ratings. Our approach aims to help automotive UI designers make better decisions about choosing the most suitable scent, as well as possible alternative modalities.
\end{abstract}

\section{KEYWORDS}

Olfaction; Smell; Mapping; Odour Stimulation; Automotive User Interfaces; Multimodal Interfaces; In-Car Interaction 
${ }^{1}$ https://www.mercedes-benz.com/ en/mercedes-benz/innovation/ a-fragrance-for-the-new-s-class/

${ }^{2}$ http://www.bmwblog.com/2015/07/03/

she-created-the-smell-of-the-all-new-bmw-7-series/

${ }^{3}$ https://www.bentleymotors.

com/en/world-of-bentley/

mulliner/personal-commissioning/

personalising-your-bentley.html

\section{INTRODUCTION}

Visual notifications dominate in modern vehicles, however, any distraction of the driver's visual attention on the road can have fatal consequences [17]. Sound can reduce the visual load and help the driver perceive the urgency of the warnings [8], but it can also be annoying [2] or even distracting [8]. This has stimulated the exploration of other modalities [14]. Tactile interfaces have been widely studied and have indicated, e.g. a positive effect on users' attention in safety critical environments [22], faster braking reaction times [11] in simulated driving, while also being less annoying [10] Olfactory stimulation is, still largely unexplored in automotive contexts, even though it could help drivers process information [20].

Olfactory stimulation is the most challenging communication channel to apply in the car, due to scent lingering and interpersonal differences [7]. It has been proven to have a positive impact on the alertness and mood of the driver [3, 19], drivers' braking performance [13], and on keeping drowsy drivers awake $[9,15,24]$. Nevertheless, there are still only very few investigations of using smell as a communication channel $[5,6]$. However, considering the increased visual load in modern infotainment and driving assistance systems, coupled with advances in scent-delivery $[1,4,12,16]$, we see a great opportunity to rethink the integration of scent into modern vehicles. A valid approach is necessary to decide what scent matches a specific driving-relevant notification. Our paper proposes the first steps on the way of establishing such a framework.

The significance and the originality of our approach can be summarised as follows:

- We propose the first approach to help automotive UI designers make better decisions about choosing the best scent for a specific driving-relevant notification. This is especially important considering the recent olfactory interface tendencies in the automotive industry, involving such manufacturers as Mercedes-Benz ${ }^{1}, \mathrm{BMW}^{2}$, and Bentley ${ }^{3}$.

- Our approach goes beyond the most relevant previous work in this area. We offer a structured method of choosing the scent for an in-car interaction scenario, by not only taking into consideration the knowledge on the effects of scents from psychology and neuroscience, but also by comparing their efficiency opposed to other modalities (i.e. vision, audition, and touch).

To make sure our method is valid, we built up on the work of Politis et al. [18], who compared different types of visual, auditory, and tactile stimulation as stand-alone and combined notifications to convey driving-relevant information. We extend this work by including the olfactory modality.

\section{METHOD}

Based on the related work, we propose establishing a framework for validating the matching between scents and notifications (by extending the approach of [18]). To do that, we focus only on one scent and one notification, which can be conveyed by this scent (multiple scents could be explored for each 


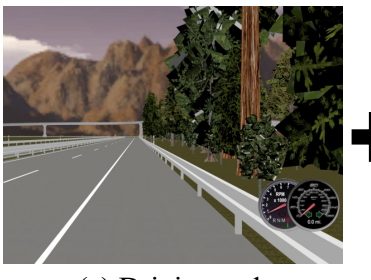

(a) Driving task

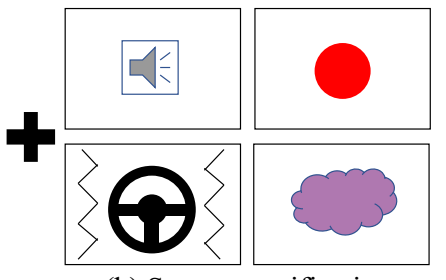

(b) Sensory notification

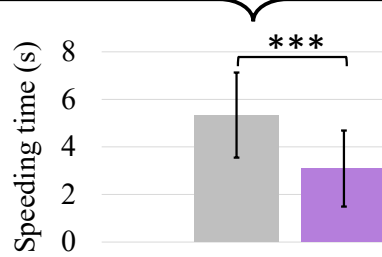

Olfaction Off $₫$ Olfaction On

(c) Analysis of the driving behaviour

Figure 1: Framework for validating the matching between driving-relevant notifications (e.g. "Slow down") and scents (e.g. Lavender). We propose a framework with the following three steps: (a) Selecting a driving task, (b) Displaying a notification (auditory, visual, tactile, olfactory, or combined) relevant to this driving task (e.g. one sensory modality/combination of modalities per condition), and (c) Analysing the driving behaviour based on what is required by the selected task (e.g. mean speeding time in seconds, in case of a "Slow down" notification, error bars $\pm \mathrm{SD}, * * * \boldsymbol{p}<.001)$. notification in the future). To demonstrate our approach, we chose a speeding scenario. The driving task involves overtaking slower vehicles. As there are also oncoming vehicles and pedestrians involved, the participant is likely to go over the speed limit when overtaking. Once this happens, the corresponding "Slow down" notification is displayed as a beep or a circular red symbol (as per [18]), as a vibration on the steering wheel (as per [21]), as a puff of Lavender (a calming and sharp scent associated with slowing down in $[5,6,23]$ ), or as a puff of clean air (control stimulus). Each of these modalities could be explored as a separate condition in a within- or a between-participants study, depending on the driving task (e.g. short task would enable a within-participants study). To extend the knowledge on multimodal interaction, it is also possible to include combined e.g. visual-olfactory, auditory-olfactory, and visual-auditory-olfactory conditions. Our framework is schematically displayed in Figure 1.

We suggest evaluating the matching based on the changes of the driving behaviour (e.g. time required to reduce the speed back to the limit, number of speeding events, mean speed, driving time, braking intensity) and performance (lane deviation). In addition to this data, we also encourage collecting the self-report data on the perceived liking and comfort of interacting with each modality.

\section{PRELIMINARY STUDY}

For an initial exploration, we conducted a preliminary study to see if a Lavender olfactory notification has a positive impact on the slow down time, mean number of speeding events, the mean speed, and the lane deviation. In this study, the driving task included no other traffic and participants were instructed to stay as close to the speed limit as possible, while driving on a motorway.

\section{Study Design}

This study followed a 1 (scent: lavender) $\times 2$ (conditions: scent vs no scent $) \times 2$ (repetitions) withinparticipants experimental design, composed of two main steps: (1) Familiarisation with the driving simulator, (2) Driving with or without a scent notification delivered every time the speed limit $(70 \mathrm{mph} / 112.654 \mathrm{~km} / \mathrm{h})$ is exceeded (i.e. two repetitions with a scent and two without). The conditions were randomised and counterbalanced using the Latin square.

\section{Setup and Procedure}

For this study, we have assembled and used the scent-delivery device and the olfactory interaction room (made of materials that do not absorb scents, equipped with an air extractor) proposed in [4] To create a feeling of being seated in a real vehicle, we have used a driving simulator seat from FK Automotive, with the Logitech G27 steering wheel mounted on it. We have used the OpenDS driving simulator software displayed on a 55 " curved screen with $60 \mathrm{~Hz}$ refresh rate. The source code of this software (in Java) was integrated with functions that we wrote to control the scent-delivery device. 


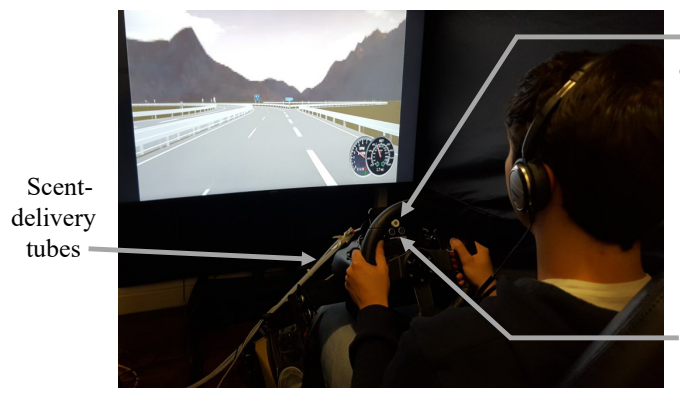

Figure 2: Participant sitting in the driving simulator located inside the olfactory interaction space
The output of the scent-delivery device was located behind the steering wheel and pointed towards the participants' face (as in [6]). The distance from the output to the face depended on how each participant adjusted their seat. We measured this distance using an ultrasound sensor located just under the scent-delivery nozzle and the mean distance among all participants was $48.95 \mathrm{~cm}(S D=6.52)$ Participants wore headphones playing the engine sound, which was cancelling any potential sounds elicited by the scent-delivery $(30 \mathrm{~dB})$ or the noise around the experimental space (see Figure 2 ).

In two trials out of four, the scent of lavender was delivered to the participants' noses every time the driving speed reached $72 \mathrm{mph}(115.873 \mathrm{~km} / \mathrm{h})$ or more. The tolerance of $2 \mathrm{mph}(3.219 \mathrm{~km} / \mathrm{h})$ was introduced to avoid potential frustration caused by going above the limit insignificantly.

In the other two trials, there were no olfactory notifications involved, and the participants were instructed to rely on the speedometer visualised on the bottom-right corner of the screen to check if they were not above the speed limit (just like drivers do on the real road). Before each trial, participants were instructed about which notifications they would receive and in what situations.

Every trial finished automatically when the participants had driven one full lap, which took 2-3 minutes. The trial was restarted in case of a crash (only one participants crashed in one of their trials).

The experiment finished with a questionnaire on the overall experience and the demographic data of the participants, followed by the debriefing. Overall, the study took about 30 minutes. This study was approved by Ethics Committee of the University of Sussex.

\section{Results}

Participants. A total of 21 participants, with a mean age of 31.05 years ( $S D=6.30,10$ females) volunteered for this study. Their mean driving experience was 10.17 years $(S D=6.16)$. Participants have reported having no olfactory dysfunctions, adverse reactions to strong scents, respiratory problems, or flu, and not being pregnant. All participants expressed their written consent before the start of the experiment.

Driving Data. We have performed a two-way repeated measures ANOVA test to compare the driving data collected with and without the olfactory notifications presented to the participants to indicate the speeding event. We will call these conditions: "olfaction on" and "olfaction off" modes.

When receiving olfactory notifications, participants have reduced the speed significantly faster $(F(3,18)=10.519, p<.001$; Wilks' $\lambda=.363)$ than without such notifications. It took them $M=5.34 s(S D=$ 1.79 ) to return the car's current speed back to the speed limit in the "olfaction off" mode, but only $M=$ $3.09 s(S D=1.60)$ in the "olfaction on" mode (see Figure 1c).

The participants have also exceeded the speed limit fewer times in the "olfaction on" $(M=3.25, S D=$ 1.58) than in the "olfaction off" $(M=4.03, S D=.90)$ mode (see Figure 3a), which was a statistically significant difference $(F(3,18)=3.304, p<.05 ;$ Wilks' $\lambda=.645)$. 


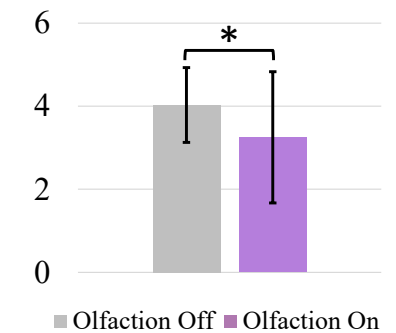

(a) Mean number of speeding event

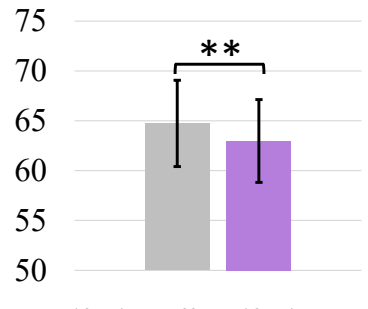

Olfaction Off $₫$ Olfaction On

Figure 3: Driving behaviour data of the "olfaction off" (no olfactory notifications) and the "olfaction on" (with olfactory notifications) conditions: (a) Mean number of times the participants have exceeded the speed limit in each trial, (b) Mean speed in mph ("olfaction off": $M=104.17 \mathrm{~km} / \mathrm{h}(S D=$ $6.97 \mathrm{~km} / \mathrm{h}$ ); "olfaction on": $M=101.34 \mathrm{~km} / \mathrm{h}$ $(S D=6.70 \mathrm{~km} / \mathrm{h})$. Error bars, $\pm \mathrm{SD}, * p<.05$ $* * \boldsymbol{p}<.01$

To assess the participants' driving performance, we have captured the lane deviation (distance from the centre of the lane in $\mathrm{cm})$. In the "olfaction on" mode, it was lower $(M=35.80 \mathrm{~cm}, S D=6.40)$ than in the "olfaction off" mode $(M=37.74 \mathrm{~cm}, S D=11.02)$, but the difference was not significant.

The results also show that with the olfactory notifications, the participants drove significantly slower $(F(3,18)=6.675, p<.01$; Wilks' $\lambda=.473)$. The mean speed in the "olfaction off" mode was $M=$ $64.73 \mathrm{mph} / 104.17 \mathrm{~km} / \mathrm{h}(S D=4.33 \mathrm{mph} / 6.97 \mathrm{~km} / \mathrm{h})$, whereas in the "olfaction on" $M=62.97 \mathrm{mph} / 101.34 \mathrm{~km} / \mathrm{h}$ $(S D=4.16 \mathrm{mph} / 6.70 \mathrm{~km} / \mathrm{h})$, which can be seen on Figure $3 \mathrm{~b}$.

\section{CONCLUSION AND FUTURE WORK}

This paper is the first to propose an approach for validating a mapping between scents and drivingrelevant notifications. There have been multiple proofs of concepts demonstrating the effectiveness of olfactory stimulation in the automotive context, but none of those indicates a clear procedure for making sure the initial mapping (e.g. as in [6]) is valid considering such driving behaviour measures as lane deviation, mean speed, and the time required to recover from error. Our initial preliminary study demonstrates how such measurements can be taken into account for the validation task. In the future, we plan to carry out the study described in the Method section to perform the complete validation of the mapping between the Lavender scent and the "Slow down" notification. Our preliminary study investigated only one scent. However, our framework does not exclude exploring multiple scents to find the best match with the chosen driving-relevant notification. We also propose using a puff of clean air as a control stimulus. The study we have planed for the future, will reveal advantages and disadvantages of using other modalities (visual, auditory, and tactile) compared to the olfactory channel. We propose exploring other modalities to find driving scenarios in which olfactory notifications are most useful.

\section{ACKNOWLEDGMENTS}

This project has received funding from the ERC under the European Union's Horizon 2020 research and innovation programme under the grant agreement No 638605 and No 737576.

\section{REFERENCES}

[1] J. Amores and P. Maes. 2017. Essence: Olfactory Interfaces for Unconscious Influence of Mood and Cognitive Performance. In CHI '17. ACM, New York, NY, USA.

[2] C.L. Baldwin. 2011. Verbal collision avoidance messages during simulated driving: Perceived urgency, alerting effectiveness and annoyance. Ergonomics 54, 4 (2011).

[3] R.A. Baron and M.J. Kalsher. 1998. Effects of a Pleasant Ambient Fragrance on Simulated Driving Performance: The Sweet Smell of... Safety? Environment and Behavior 30, 4 (1998).

[4] D. Dmitrenko, E. Maggioni, and M. Obrist. 2017. OSpace: Towards a Systematic Exploration of Olfactory Interaction Spaces. In ISS '17. ACM, New York, NY, USA. 
[5] D. Dmitrenko, E. Maggioni, and M. Obrist. 2018. I Smell Trouble: Using Multiple Scents To Convey Driving-Relevan Information. In ICMI '18. ACM, New York, NY, USA.

[6] D. Dmitrenko, E. Maggioni, C.T. Vi, and M. Obrist. 2017. What Did I Sniff?: Mapping Scents Onto Driving-Related Messages. In AutomotiveUI '17. ACM, New York, NY, USA.

[7] D. Dmitrenko, C.T. Vi, and M. Obrist. 2016. A Comparison of Scent-Delivery Devices and Their Meaningful Use for In-Ca Olfactory Interaction. In AutomotiveUI '16. ACM, New York, NY, USA.

[8] J. Edworthy, S. Loxley, and I. Dennis. 1991. Improving auditory warning design: Relationship between warning sound parameters and perceived urgency. Human Factors 33, 2 (1991).

[9] H. Funato, M. Yoshikawa, M. Kawasumi, S. Yamamoto, M. Yamada, and Y. Yanagida. 2009. Stimulation effects provided to drivers by fragrance presentation considering olfactory adaptation. In Intelligent Vehicles Symposium 2009. IEEE.

[10] John D. Lee, Joshua D. Hoffman, and Elizabeth Hayes. 2004. Collision Warning Design to Mitigate Driver Distraction. In CHI '04. ACM, USA.

[11] J. Lylykangas, V. Surakka, K. Salminen, A. Farooq, and R. Raisamo. 2016. Responses to visual, tactile and visual-tactile forward collision warnings while gaze on and off the road. Transportation Research Part F: Traffic Psychology and Behaviour 40 (2016).

[12] E. Maggioni, R. Cobden, D. Dmitrenko, and M. Obrist. 2018. Smell-O-Message: integration of olfactory notifications into a messaging application to improve users' performance. In ICMI '18. ACM, New York, NY, USA.

[13] G.N. Martin and J.A. Cooper. 2007. Adding zest to difficult journeys: Odour effects on simulated driving performance. In The British Psychological Society Annual Conference.

[14] M. Obrist, A.N. Tuch, and K. Hornbaek. 2014. Opportunities for Odor: Experiences with Smell and Implications for Technology. In CHI '14. ACM, New York, NY, USA.

[15] C. Oshima, A. Wada, H. Ando, N. Matsuo, S. Abe, and Y. Yanigada. 2007. Improved delivery of olfactory stimukus to keep drivers awake. In Workshop on DSP for in-Vehicle and Mobile Systems.

[16] B. Patnaik, A. Batch, and N. Elmqvist. 2019. Information Olfactation: Harnessing Scent to Convey Data. IEEE transactions on visualization and computer graphics (2019).

[17] G.A. Peters and B.J. Peters. 2001. The distracted driver. Journal of The Royal Society for the Promotion of Health (2001).

[18] I. Politis, S. Brewster, and F. Pollick. 2015. To Beep or Not to Beep?: Comparing Abstract Versus Language-Based Multimodal Driver Displays. In CHI '15. ACM, New York, NY, USA.

[19] B. Raudenbush, R. Grayhem, T. Sears, and I. Wilson. 2009. Effects of Peppermint and Cinnamon Odor Administration on Simulated Driving Alertness, Mood and Workload. N.A.J. of Psychology (2009).

[20] A. Seigneuric, K. Durand, T. Jiang, J.-Y. Baudouin, and B. Schaal. 2010. The nose tells it to the eyes: Crossmodal associations between olfaction and vision. Perception 39, 11 (2010).

[21] G. Shakeri, A. Ng, J.H. Williamson, and S.A. Brewster. 2016. Evaluation of Haptic Patterns on a Steering Wheel. In Automotive'UI 16. ACM, New York, NY, USA.

[22] A.E. Sklar and N.B. Sarter. 1999. Good vibrations: Tactile feedback in support of attention allocation and human-automation coordination in event-driven domains. Human Factors 41, 4 (1999).

[23] P. Wintersberger, D. Dmitrenko, C. Schartmüller, A.K. Frison, E. Maggioni, M. Obrist, and A. Riener. 2019. S(C)ENTINEL Monitoring Automated Vehicles with Olfactory Reliability Displays. In IUI '19. ACM, New York, NY, USA.

[24] M. Yoshida, C. Kato, Y. Kakamu, M. Kawasumi, H. Yamasaki, S. Yamamoto, T. Nakano, and M. Yamada. 2011. Study on Stimulation Effects for Driver Based on Fragrance Presentation. In MVA2011 IAPR Conference on Machine Vision Applications. 